\title{
Skeletal Muscle
}

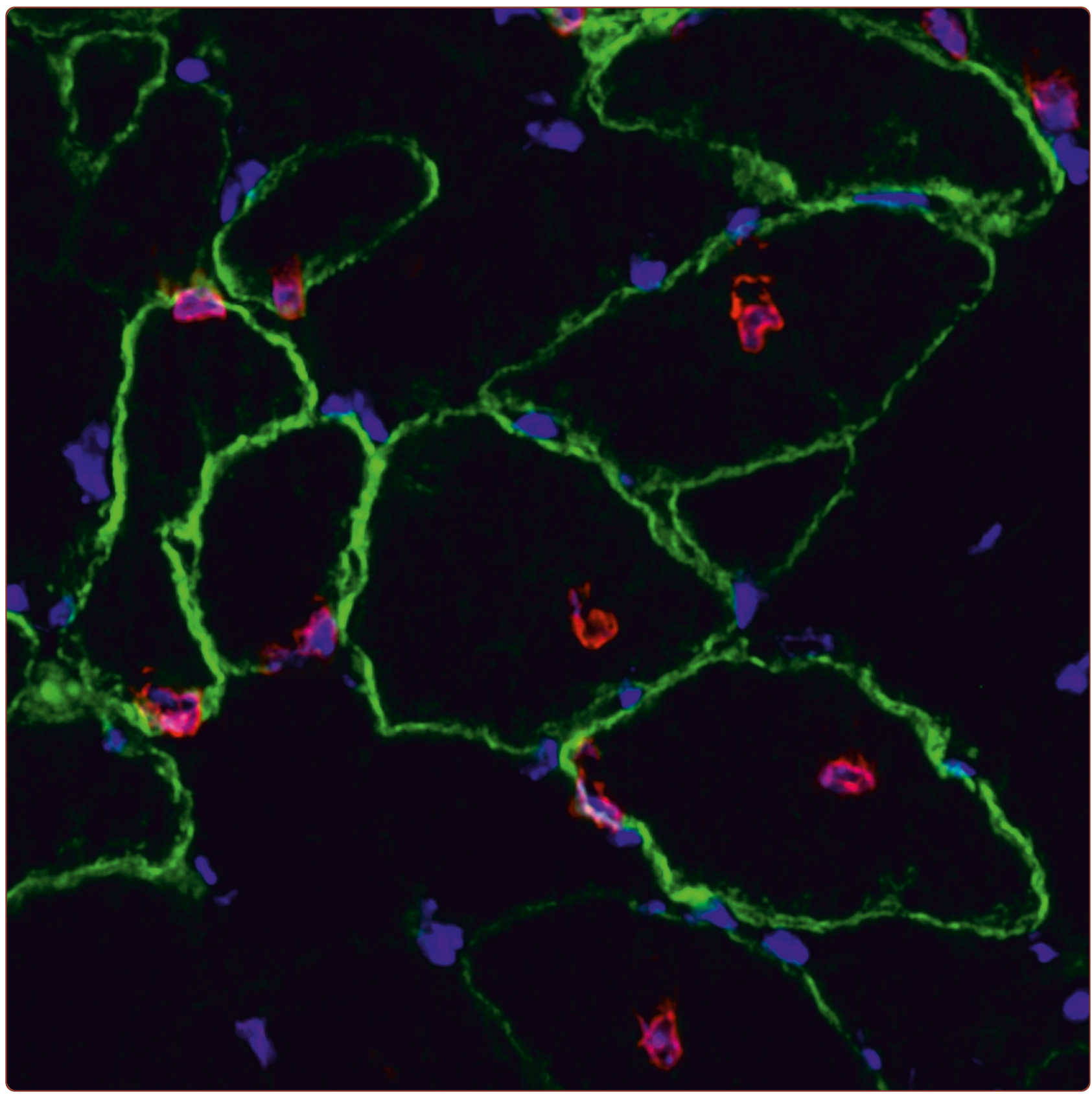

Immortalized pathological human myoblasts: towards a universal tool for the study of neuromuscular disorders

Mamchaoui et al. 


\title{
Immortalized pathological human myoblasts: towards a universal tool for the study of neuromuscular disorders
}

\author{
Kamel Mamchaoui ${ }^{1,2,3 \dagger}$, Capucine Trollet ${ }^{1,2,3+}$, Anne Bigot ${ }^{1,2,3}$, Elisa Negroni ${ }^{1,2,3}$, Soraya Chaouch ${ }^{1,2,3}$, Annie Wolff ${ }^{1,2,3}$ \\ , Prashanth K Kandalla $a^{1,2,3}$, Solenne Marie ${ }^{1,2,3}$, James Di Santo ${ }^{4}$, Jean Lacau St Guily ${ }^{1,2,3,5}$, Francesco Muntoni ${ }^{6}$, \\ Jihee Kim ${ }^{6}$, Susanne Philippi ${ }^{1,7}$, Simone Spuler ${ }^{7}$, Nicolas Levy ${ }^{8}$, Sergiu C Blumen ${ }^{9}$, Thomas Voit ${ }^{1,2,3}$, \\ Woodring E Wright ${ }^{10}$, Ahmed Aamiri ${ }^{11}$, Gillian Butler-Browne ${ }^{1,2,3}$ and Vincent Mouly ${ }^{1,2,3^{*}}$
}

\begin{abstract}
Background: Investigations into both the pathophysiology and therapeutic targets in muscle dystrophies have been hampered by the limited proliferative capacity of human myoblasts. Isolation of reliable and stable immortalized cell lines from patient biopsies is a powerful tool for investigating pathological mechanisms, including those associated with muscle aging, and for developing innovative gene-based, cell-based or pharmacological biotherapies.
\end{abstract}

Methods: Using transduction with both telomerase-expressing and cyclin-dependent kinase 4-expressing vectors, we were able to generate a battery of immortalized human muscle stem-cell lines from patients with various neuromuscular disorders.

Results: The immortalized human cell lines from patients with Duchenne muscular dystrophy, facioscapulohumeral muscular dystrophy, oculopharyngeal muscular dystrophy, congenital muscular dystrophy, and limb-girdle muscular dystrophy type $2 \mathrm{~B}$ had greatly increased proliferative capacity, and maintained their potential to differentiate both in vitro and in vivo after transplantation into regenerating muscle of immunodeficient mice.

Conclusions: Dystrophic cellular models are required as a supplement to animal models to assess cellular mechanisms, such as signaling defects, or to perform high-throughput screening for therapeutic molecules. These investigations have been conducted for many years on cells derived from animals, and would greatly benefit from having human cell models with prolonged proliferative capacity. Furthermore, the possibility to assess in vivo the regenerative capacity of these cells extends their potential use. The innovative cellular tools derived from several different neuromuscular diseases as described in this report will allow investigation of the pathophysiology of these disorders and assessment of new therapeutic strategies.

\section{Background}

Muscular dystrophies constitute a heterogeneous group of genetic muscle diseases characterized by progressive muscle weakness, wasting and degeneration, some of these features are common to muscle aging [1,2]. Over the past few years, the genetics and pathophysiology of

\footnotetext{
* Correspondence: vincent.mouly@upmc.fr

† Contributed equally

'Thérapie des maladies du muscle strié, Institut de Myologie, UM76, UPMC

Université Paris 6, Paris, France

Full list of author information is available at the end of the article
}

some of these diseases has been deciphered, stimulating the development of novel gene-based (or mRNA-based) (for example, gene therapy, exon-skipping or codon read-through), cell-based and pharmacological therapies [3], which can either target the mutation directly, or target the consequences of that mutation, such as muscle wasting, atrophy or denervation. To assess these rapidly developing therapeutic advances, there is a crucial need to develop standardized tools to determine the cellular and molecular mechanisms that trigger the physiopathologic modifications, and to assess these new therapeutic

\section{() Biomed Central}


strategies in preclinical trials. Transgenic mice have often been used to investigate the physiopathology of muscular dystrophies [4-6]; however, the mutation remains in a murine context, and there are often major differences between humans and mice; for example, a mutation in the dystrophin gene results in a mild pathological phenotype in $m d x$ mice but in a progressive and fatal disease (Duchenne muscular dystrophy; DMD) in humans. Furthermore, not every mutation can be created and evaluated in murine models, and mechanisms common to aging and dystrophies may differ between mice and humans. Consequently, human primary myoblasts isolated from dystrophic patient biopsies provide the most pertinent experimental models to assess a variety of human genetic mutations in their natural genomic environment. Although in vitro models do not fully recapitulate the in vivo environment, cell-culture systems allow rapid, high-throughput screening of molecules or oligonucleotides, and new strategies can be easily tested prior to validation in animal models, which is a costly and time-consuming process. The main drawbacks of using in vitro primary cultures of human cells derived from muscle biopsies are their purity, their limited proliferative capacity, and the variation in phenotype when amplified in vitro; their phenotype will always be confounded by modifications due to cellular senescence, which will progressively occur during cell amplification $[7,8]$.

The two major mechanisms responsible for this replicative cellular senescence seen in human myoblasts are (i) activation of the p16-mediated cellular stress pathway, and (ii) the progressive erosion of telomeres at each cell division until they reach a critical length that will trigger p53 activation and cell-cycle exit $[9,10]$. Introduction of the telomerase catalytic subunit (human telomerase reverse transcriptase; $h T E R T)$ cDNA alone will result in an extension of the lifespan and even immortalization in a variety of cell types, including endothelial cells and fibroblasts $[11,12]$. However, we have shown that the expression of both hTERT and cyclin-dependent kinase (CDK)-4 is required to successfully overcome cellular senescence in human myoblasts [13]; while hTERT elongates the telomere, CDK-4 blocks the $\mathrm{p} 16^{\mathrm{INK} 4 \mathrm{a}}$-dependent stress pathway.

In the present study, our goal was to create a large collection of immortalized human myoblasts isolated from a wide range of neuromuscular disorders (DMD, facioscapulohumeral muscular dystrophy (FSHD), oculopharyngeal muscular dystrophy (OPMD), limb-girdle muscular dystrophy (LGMD2B or dysferlinopathy) and congenital muscular dystrophy (CMD)), which could be used as experimental tools to study these diseases and to develop new therapeutic strategies.
DMD is the most common childhood muscular dystrophy. It is caused by mutations in the dystrophin gene encoding an essential protein of the muscle membrane cytoskeleton [14], leading to rapid and progressive skeletal-muscle weakness. FSHD is a progressive muscle disease caused by contractions in a $3.3 \mathrm{~kb}$ repeat region (D4Z4) located at 4q35.2 [15], which first affects the muscles of the face and upper limb girdle with asymmetry, and later the lower limb girdle. OPMD is a rare, autosomal dominant, late-onset degenerative muscle disorder caused by a short $(\mathrm{GCG})_{\mathrm{n}}$ triplet expansion in the poly $(A)$ binding protein nuclear 1 (PABPN1) gene [16], which affects the eyelid and pharyngeal muscles. LGMD2B is a recessive muscle disease caused by mutations in the dysferlin gene, a muscle membrane protein known to be involved in membrane repair [17] and trafficking. The disease is characterized by early and slowly progressive weakness and atrophy of the pelvic and shoulder girdle muscles in early adulthood. Finally, CMD refers to a clinically and genetically heterogeneous group of dystrophies, which result in the onset of muscle weakness at birth or in childhood, and involve mutations in several proteins such as collagen, laminin, integrin, and nesprin 1 [18].

In this study, we report for the first time that for each of these muscular dystrophies, we were able to produce reliable and stable immortalized cell lines from human myoblasts isolated from biopsies, resulting in robust in vitro models that can also be implanted in vivo. This non-exhaustive list of cellular models will provide powerful and valuable tools for the scientific community investigating these pathological conditions and/or their mechanisms. as they overcome the problem of limited proliferation usually present in myoblasts. These models should also be useful in the development of gene or cell therapies and pharmacological strategies for muscular dystrophies, some of which might also be used to combat muscle weakness in the elderly.

\section{Methods}

\section{Ethics approval}

Muscle biopsies (Table 1) were obtained from the BTR (Bank of Tissues for Research, a partner in the EU network EuroBioBank) or from neurologists, in accordance with European recommendations and French legislation. Surgical procedures were performed in accordance with the legal regulations in France and European Union ethics guidelines for animal research.

\section{Human myoblast cultures}

Human myoblasts were isolated from biopsies and cultivated as described previously [19] in a growth medium consisting of 199 medium and DMEM (Invitrogen Carlsbad, CA) in a 1:4 ratio, supplemented with $20 \%$ 
Table 1 Muscle biopsies obtained from various neuromuscular dystrophies.

\begin{tabular}{lllll}
\hline Name & Disease & Genetic defect & Donor muscle & Age \\
\hline CTRL & None & None & Semitendinosus & 25 years \\
\hline CMD & Congenital muscular dystrophy & $2345 \mathrm{G}>$ T; nesprin-1 gene & Paravetrebral & 16 years \\
\hline DMD & Duchenne muscular dystrophy & Deletion of exon 48-50; dystrophin gene & Quadriceps & 20 months \\
\hline FSHD & Fascioscapulohumeral muscular dystrophy & 2 D4Z4 contraction & Subscapularis & 27 years \\
\hline LGMD2B & Limb-girdle muscular dystrophy type 2B & 1448C $>$ A and 107T $>$ A; dysferlin gene & Quadriceps & 40 years \\
\hline OPMD & Oculopharyngeal muscular dystrophy & Expansion $(G C G)_{9}-(G C G)_{6 ;}$ PABPN1 gene & Cricopharyngeal & 60 years \\
\hline
\end{tabular}

FCS (Invitrogen), $2.5 \mathrm{ng} / \mathrm{ml}$ hepatocyte growth factor (Invitrogen), $0.1 \mu \mathrm{mol} / \mathrm{l}$ dexamethasone (Sigma-Aldrich, St. Louis, MO, USA) and $50 \mu \mathrm{g} / \mathrm{ml}$ gentamycin (Invitrogen). The myogenic purity of the populations was monitored by immunocytochemistry using desmin as marker. Enrichment of myogenic cells was performed using an immunomagnetic cell sorting system (MACS; Miltenyi Biotec, Paris, France) according to the manufacturer's instructions. Briefly, cells were labeled with anti-CD56 (a specific marker of myoblasts) microbeads, and then separated in a MACS column placed in a magnetic field. Purification was checked by immunochemistry using a desmin marker. Differentiation was induced at confluence by replacing the growth medium with DMEM supplemented with $100 \mu \mathrm{g} / \mathrm{ml}$ transferrin, $10 \mu \mathrm{g} / \mathrm{ml}$ insulin and $50 \mu \mathrm{g} / \mathrm{ml}$ of gentamycin (Sigma-Aldrich).

\section{Cell transduction}

$h T E R T$ and $C d k 4$ cDNA were cloned into different pBABE retroviral vectors containing puromycin and neomycin selection markers, respectively. Infection was carried out as described previously [20]. Transduced cell cultures were selected with puromycin $(0.2 \mu \mathrm{g} / \mathrm{ml})$ and/ or neomycin $(0.3 \mathrm{mg} / \mathrm{ml})$ for 8 days. The infected cells were purified as described previously if necessary, and were then seeded at clonal density. Selected individual myogenic clones were isolated from each population, using glass cylinders, and their proliferation and differentiation capacities were characterized.

\section{Telomere length analysis}

Genomic DNA was extracted from each proliferating cell line using a salting-out procedure. Telomere length was determined by using a quantitative (q)PCR method, as previously described $[21,22]$. PCR amplification was achieved using telomere $(\mathrm{T})$ and single-copy gene 36B4 (acidic ribosomal phosphoprotein P0) (S) primers. The mean telomere length was calculated as the ratio of telomere repeats to $36 \mathrm{~B} 4$ copies, represented as the $\mathrm{T}: \mathrm{S}$ ratio. Each sample was run in triplicate, using $20 \mathrm{ng}$ of DNA per replicate, and three independent runs were analyzed. The primer sequences and detailed PCR protocols used are available on request.

\section{Reverse transcriptase PCR}

To analyze the expression of myogenic markers in proliferating primary and immortalized cell lines, $1 \mu \mathrm{g}$ RNA from each cell line was used for the cDNA synthesis (Superscript III; Invitrogen) using random hexamer primers. cDNA $(1 \mu \mathrm{l})$ was used as a template for PCR using N-CAM, MyoD and desmin specific primers. The primer sequences and detailed PCR protocols used are available on request.

\section{Induction of host muscle regeneration and implantation} of human cells

Immunodeficient $\mathrm{Rag} 2^{-1-} \gamma \mathrm{C}^{-1-} \mathrm{C}^{-1-}$ mice aged 2 to 3 months were anesthetized with an intraperitoneal injection of ketamine hydrochloride $(80 \mathrm{mg} / \mathrm{kg})$ and xylasin $(10 \mathrm{mg} / \mathrm{kg})$ (Sigma-Aldrich). To induce severe muscle damage and trigger regeneration, the recipient tibialis anterior (TA) muscles were exposed to cryodamage, and a single injection of immortalized human cells $(15 \mu \mathrm{l}$ of cell suspension containing $2.5 \times 10^{5}$ or $5 \times 10^{5}$ cells in PBS) was administered as described previously [23]. Four weeks after transplantation, the recipient TA muscles were dissected, mounted in gum tragacanth, and frozen in liquid nitrogen-cooled isopentane for later analysis.

\section{Immunofluorescence}

In vitro and in vivo characterizations were performed by immunolabeling as described previously [23-25]. Antibodies used were directed against myosin isoforms (MF20, mouse IgG2b, 1:20 dilution; Developmental Studies Hybridoma Bank, DSHB, Iowa City, IA), lamin A/C (clone JOL2, mouse IgG1, 1:300; AbCam, Cambridge, Cambridgeshire, UK), lamin A/C (NCL-LAM A/C, clone 636, mouse IgG2b, 1:400, Novocastra, Newcastle-uponTyne, Tyne and Wear, UK), spectrin (NCL-Spec1, clone RBC2/3D5, mouse IgG2b, 1:50; Novocastra), and laminin (rabbit polyclonal, Z 0097, 1:400; Dako, Trappes, France). The secondary antibodies used were Alexa Fluor 488-conjugated goat anti-mouse IgG2b (Molecular Probes, Montluçon, France), Alexa Fluor 647-conjugated goat anti-rabbit (Molecular Probes), and Cy3-conjugated goat anti-mouse IgG1 (Jackson Immunoresearch, West 
Grove, PA, USA). Images were visualized using a microscope (Olympus Corp., Tokyo, Japan), and digitized using a charge-coupled device (CCD) camera (Olympus Corp., Tokyo, Japan).

\section{Antisense oligonucleotides transfection and reverse transcriptase PCR}

Cells were seeded in six-well plates and grown in growth medium. Transfection of antisense oligonucleotides (AONs) was performed using $1 \mu \mathrm{l}$ of transfection reagent (Lipofectamin 2000; Invitrogen) per $\mu \mathrm{g}$ of AONs for 4 hours. The chemistry used for AONs was 2'-Omethyl-phosphorothioates. All transfections were performed with at least two independent duplicates. Cells were changed to differentiation medium before transfection. Typically 24 to 48 hours after transfection, RNA was extracted from the cells using Trizol or Qiagen column kit (Qiagen Inc., Valencia, CA, USA). 1 \% RNA was used for the cDNA synthesis (Superscript III; Invitrogen) with DMD exon-specific primers. cDNA $(2 \mu \mathrm{l})$ was used as a template for a first PCR reaction. From this first reaction of 25 cycles, $1 \mu \mathrm{l}$ of the product was removed and used as a template for a second nested PCR of 35 cycles. PCR products were analyzed on 1.5 to $2 \%$ agarose gels. The primer sequences and detailed PCR protocols used are available on request.

\section{Results}

Immortalized myoblast lines generated from dystrophic muscles

Primary cultures from distinct muscular dystrophies (DMD, FSHD, OPMD, CMD and LGMD2B, Table 1) were co-transduced with two retroviral vectors expressing hTERT and CDK-4 cDNA. Co-transduced cells were selected by neomycin and puromycin and then purified using magnetic beads coupled to antibodies directed against the myogenic marker CD56. Following culture at clonal density, individual myogenic clones with extended proliferative lifespans, as compared to the untranduced cells, were isolated from each population. In contrast to the parental populations, which stopped proliferating at various stages of the culture, depending on the type of dystrophy, the selected immortalized clones were still able to proliferate after prolonged amplification in vitro under the same culture conditions (Figure 1). All immortalized clones were cultivated until they had achieved at least twice as many divisions as the parental population.

Telomere length was measured in each clone (Table 2) and ranged from $10.3 \mathrm{~kb}$ to $24.8 \mathrm{~kb}$ with no difference between the clones and control immortalized myoblasts $(17.6 \mathrm{~kb})$. The length of the telomeres in all of the immortalized myogenic clones was always well above the 6 to $7 \mathrm{~kb}$ limit usually seen in control cells that are reaching senescence, but remained within the range of that seen both in myoblasts and in other stem cells (12 to $20 \mathrm{~kb})$.

\section{In vitro characterization of immortalized cells}

To confirm that the immortalized cell lines maintained their myogenic signature, we compared the expression of several markers in proliferating primary and immortalized cell lines from control, OPMD and DMD biopsies. In all of them, we confirmed the expression of the myogenic markers desmin, neural cell adhesion molecule (N-CAM) and MyoD (Figure 2A).

In addition, we also tested their ability to differentiate into myotubes, using immunostaining with MF20 antibody, which recognizes all skeletal-muscle myosin heavy chains (MyHCs). After 5 days in differentiation conditions, all of the immortalized cell lines were able to fuse into myotubes expressing MyHCs (Figure 2B).

\section{Induction of host muscle regeneration and implantation of human cells}

To investigate the in vivo behavior of these immortalized cells, cells were grafted into damaged TA muscles of Rag2 $2^{-/-} \gamma \mathrm{C}^{-/-} \mathrm{C}^{-/-}$mice; injected muscles were analyzed 4 weeks after transplantation, which corresponds to a complete fiber regeneration process, using antibodies specific for human lamin $\mathrm{A} / \mathrm{C}$ (expressed in all human nuclei) and human spectrin (expressed in differentiated fibers). For each injected clone (control, DMD, FSHD, OPMD, CMD or LGMD2B), mature muscle fibers containing human spectrin protein and human lamin $\mathrm{A} / \mathrm{C}+$ nuclei were seen (Figure 3A). No tumors were ever observed in these immunodeficient mice.

Using antibodies specific for the basal lamina protein laminin, lamin $\mathrm{A} / \mathrm{C}$ (human nuclei) and spectrin (specific to the human protein) to identify fiber sarcolemma, we investigated if these cell lines could replenish the muscle stem-cell niche (allowing self-renewal), at the periphery of the muscle fiber and beneath the basal lamina. Whereas the vast majority of the lamin $\mathrm{A} / \mathrm{C}$ positive nuclei (97\%) were found as myonuclei (upper panel, Figure 3B), we observed the unexpected finding that all the human cells outside the muscle fibers were present in the interstitial space, separated from the fibers by a basal lamina (lower panel, Figure 3B), and not in the satellite-cell niche, suggesting that the immortalized cells were engaged preferentially in the differentiation pathway and not in the self-renewal process.

\section{Immortalized cell lines as a useful tool for therapeutic preclinical studies}

To show that these cell lines could be powerful tools to develop therapeutic strategies, we used them to evaluate 


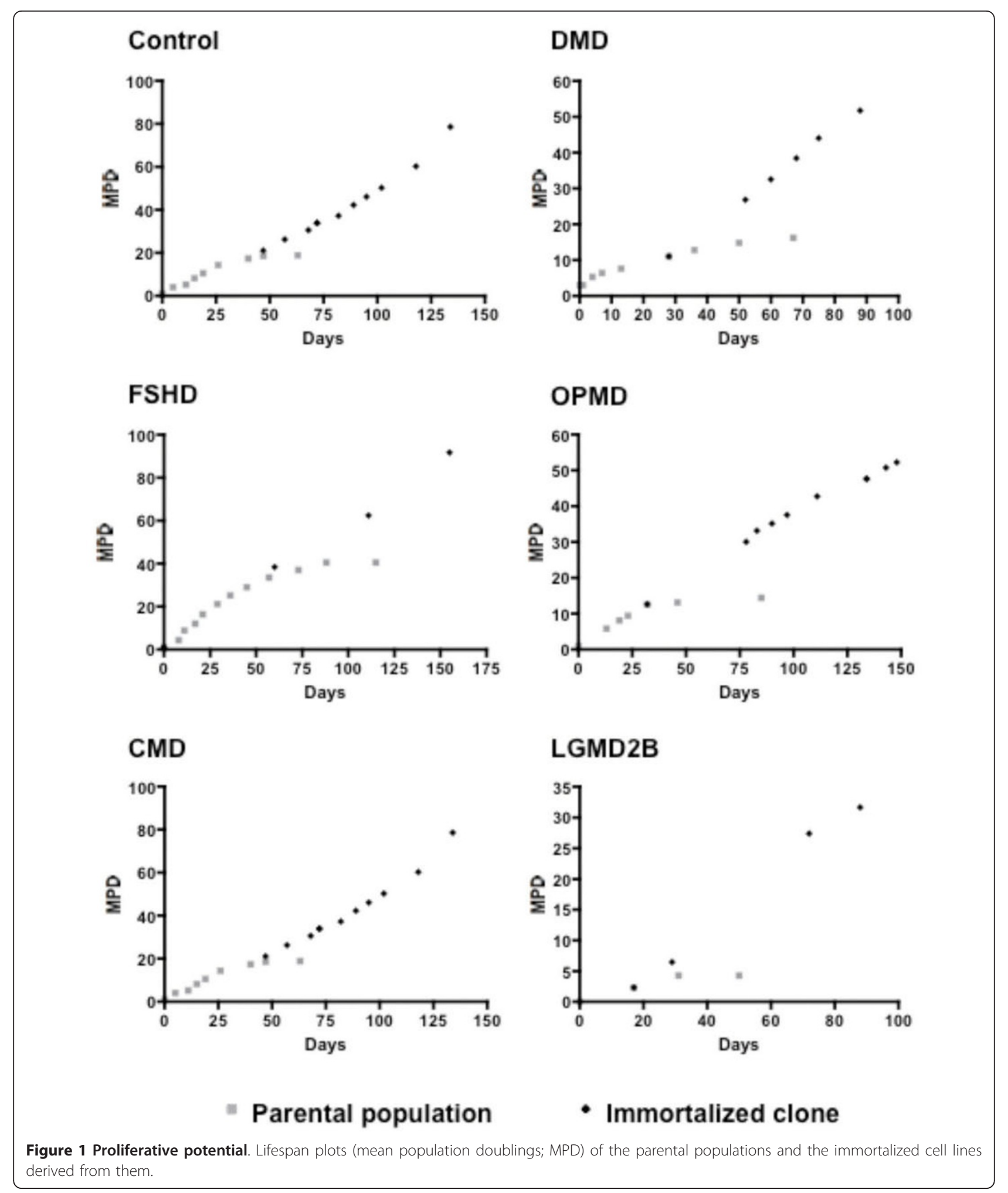

the efficiency of AONs in an exon-skipping strategy for DMD. The first one tested was AO51, which resulted in efficient skipping of exon 51 using both the primary and immortalized DMD cell lines ( $\Delta 48$ to 50 ; Figure $4 \mathrm{~A}$ ) and this AON is currently being used in a phase I/II clinical trial. Using immortalized control cells, we were able to screen a range of AONs targeting exons 17, 18, $21,22,43,44$ and 45 of the dystrophin gene. RT-PCR 
Table 2 Mean telomere length of control and immortalized cell lines.

\begin{tabular}{llll}
\hline Name & Clone & $\begin{array}{l}\text { Number of } \\
\text { divisions }\end{array}$ & $\begin{array}{l}\text { Telomere length, kb, mean } \\
\pm \text { SEM }\end{array}$ \\
\hline CTRL & C25Cl48 & 127 & $17.6 \pm 0.3$ \\
\hline CMD & CMDCl12 & 42 & $20.8 \pm 1.7$ \\
\hline DMD & DMDCl2 & 57.9 & $10.3 \pm 0.1$ \\
\hline FSHD & FSHDCl17 & 37.9 & $24.8 \pm 1.6$ \\
\hline LGMD2B & LGMD2Cl11 & 27.4 & $17.2 \pm 3.0$ \\
\hline OPMD & OPMDCl2 & 47.6 & $20.0 \pm 0.5$ \\
\hline
\end{tabular}

CMD, congenital muscular dystrophy; DMD, Duchenne muscular dystrophy; FSHD, fascioscapulohumeral muscular dystrophy; LGMD2B, limb-girdle muscular dystrophy type 2B; OPMD, oculopharyngeal muscular dystrophy

identified efficient skipping for two of them (Figure 4B, and data not shown).

\section{Discussion}

Immortal human cell lines, as long as they retain their capacity to express a specific program, are essential to study cellular and molecular mechanisms (as exemplified by the number of studies conducted on the mouse cell line $\mathrm{C} 2 \mathrm{C} 12$ ), and responses to potential therapeutic strategies. The relatively short proliferative lifespan of human myoblasts, reduced even more in dystrophic conditions by successive cycles of degeneration/regeneration in vivo prior to isolation and the modification in their myogenic potential as they approach senescence [26-28], limits their potential use. As a consequence, any assessment of pathological mechanisms or of therapeutic strategies will be biased by the presence of senescent cells, which will modify the behavior of the population. This is even more crucial for high-throughput screening of molecules, for which large numbers of cells are required. Immortalization can solve this problem, as long as the cell lines are stable and retain most of the characteristics of the unmodified parental population. This has been shown to be a problem with the $\mathrm{C} 2 \mathrm{C} 12$ cell line, as the phenotype drifts and therefore can vary both within and between different laboratories.

Immortal cell lines have been generated from human skeletal muscle, such as those derived from rhabdomyosarcomas, a rare form of skeletal-muscle tumor. However, these lines often have impaired fusion characteristics and perturbed myogenic programs $[29,30]$. Other approaches have used transduction of the large $\mathrm{T}$ antigen from SV40, which binds $\mathrm{Rb}$ and $\mathrm{p} 53$, but does not stop telomere shortening. Although these cells do have an extended lifespan, they are not immortal, and extensive telomeric erosion results in an increased frequency of chromosomal rearrangement [31] and a defective differentiation program [32]. More recently, cell lines were generated by the transduction of primary myoblasts with both hTERT and Bmi-1, which
A.

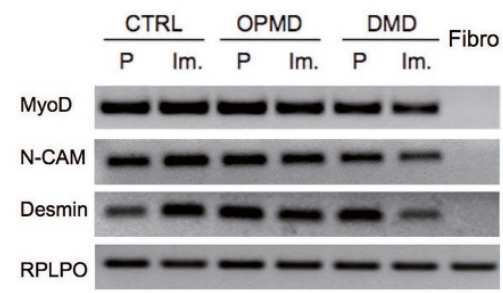

B.

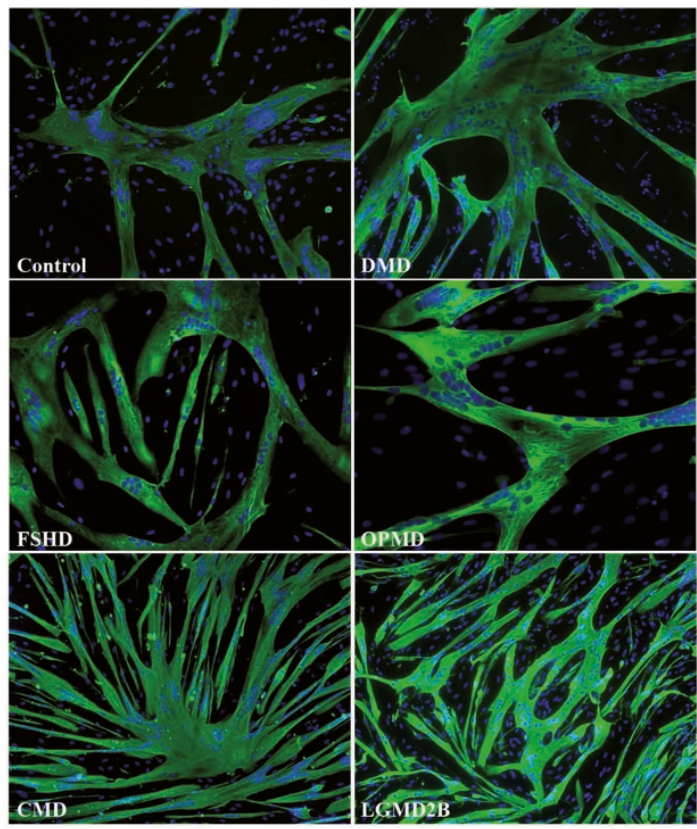

Figure 2 Myogenic markers and in vitro differentiation. (A) Reverse transcriptase PCR comparing primary $(\mathrm{P})$ and immortalized (Im.) cell lines (control, oculopharyngeal muscular dystrophy (OPMD), Duchenne muscular dystrophy (DMD)) for myogenic markers (MyoD, N-CAM and desmin). Fibroblasts were used as a negative control. (B) Immunofluorescence was carried out using MF20, an antibody directed against sarcomeric myosin (green) after 5 days of differentiation. Specific antibody labeling was visualized using Alexa Fluor 488 secondary antibody (green). Nuclei were visualized with Hoechst (blue). Original magnification $\times 100$.

downregulates both the p16 and p19Arf tumor suppressor genes, encoded by the Ink4 locus. These cells had an extended lifespan with no chromosomal rearrangement, but the differentiation potential of control myoblasts was found to be impaired [33]. This year, a report described the same approach as we have used in the present study (introduction of hTERT and CDK4) using muscle cells isolated from patients affected with FSHD [34]. The FSHD mutation causes a defect within myogenic cells, thus the establishment of a clonal myogenic cell line permit reproducible study of the consequences 


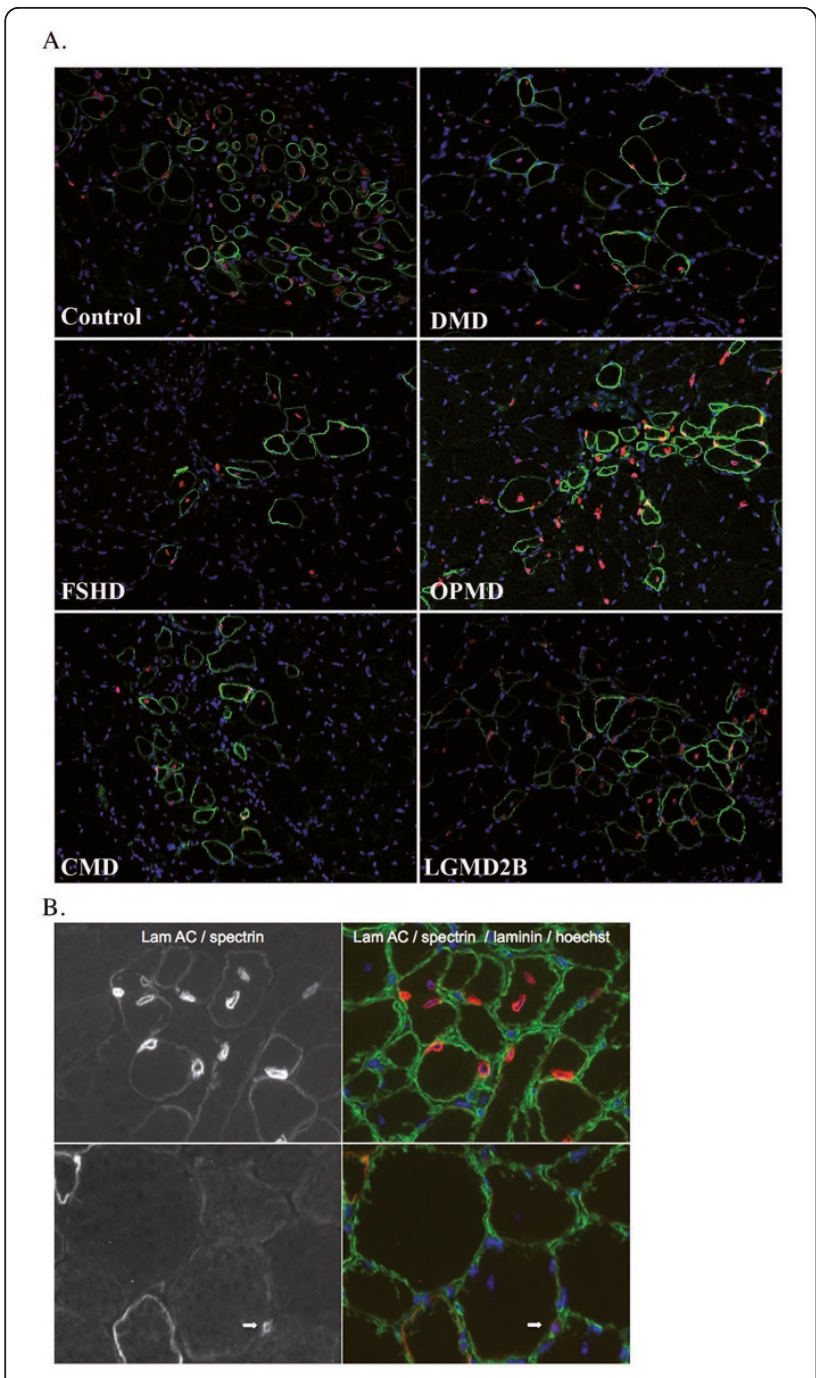

Figure 3 Detection of human immortalized cells injected into in the tibialis anterior muscle of Rag2 ${ }^{-/-} \mathrm{CC}^{-/-} \mathrm{C}^{-/-}$mice. (A) Human nuclei were visualized using an anti-lamin A/C antibody (red) and fibers expressing human proteins were visualized using an anti-human spectrin-specific antibody (green). Nuclei are counterstained with Hoechst. Original magnification $\times 200$. (B) The immortalized cells were present preferentially as myonuclei (top panel), with a small number found in interstitial space (arrow bottom panel), identified by human lamin expression (laminin staining in green, laminA/C and spectrin staining in red, Hoechst in blue, on the LGMD2B muscle section as an example). Original magnification $\times 600$.

of this mutation within myoblasts without the contamination of non-myogenic cells. The decrease in myogenicity in the primary culture, and consequently the enrichment of non-myogenic cells during amplification, is a considerable problem in the study of dystrophy diseases. For example, in 2006, we described rapid loss of myogenicity during successive passages of primary cultures of muscle cells isolated from patients with OPMD [35]; many muscle diseases are subject to a similar decrease in myogenicity. In this report, we describe the isolation of immortalized human myoblast lines from a wide range of neuromuscular diseases, using a combination of hTERT and CDK-4. This wide range of diseases paves the way to searching for common mechanisms between distinct dystrophies, and even those shared with muscle aging, as opposed to those mechanisms specific for each disease. We found that these cell lines have extended proliferative lifespans, and maintain their capacity to differentiate both in vitro and in vivo after transplantation into the regenerating muscles of immunodeficient mice. We found that these human myoblast cell lines expressing myogenic markers could colonize the host muscles and form mature fibers, thus providing an ideal model to assess therapeutic strategies in vivo, which is closer to bona fide differentiation of muscle stem cells than the converted fibroblasts described previously [36]. We also found that these cells do not replenish the muscle stem-cell niche as primary human myoblasts do under the same conditions of implantation [23], but are engaged primarily in the differentiation pathway. The process of immortalization involves overexpression of both hTERT and CDK-4, and further investigations will be needed to analyze how this may influence the balance between self-renewal and differentiation.

\section{Conclusions}

These human myoblast lines represent a powerful tool to assess signaling and/or functional deregulations in neuromuscular diseases, particularly those in which these mechanisms have not yet been clearly elucidated (FSHD, OPMD) or those with features common to muscle aging, such as atrophy or muscle wasting. We have described only a subset of the cell lines produced; we have now generated more than 35 cell lines with various mutations covering a range of 14 different pathologies, as well as cell lines from control subjects of various ages. The in vivo implantation of these cells offers the possibility to investigate the consequences of defined mutations on cellular behavior in vivo, particularly with regard to their regenerative capacity. Finally, the development of therapeutic strategies, whether these strategies imply gene, cell or pharmacological therapy involving high-throughput screening, is facilitated using these tools before assessment in animal models. As an example, we have described a rapid screen of a range of AONs in an exon-skipping strategy for DMD. In conclusion, the co-transduction of hTERT and CDK4 


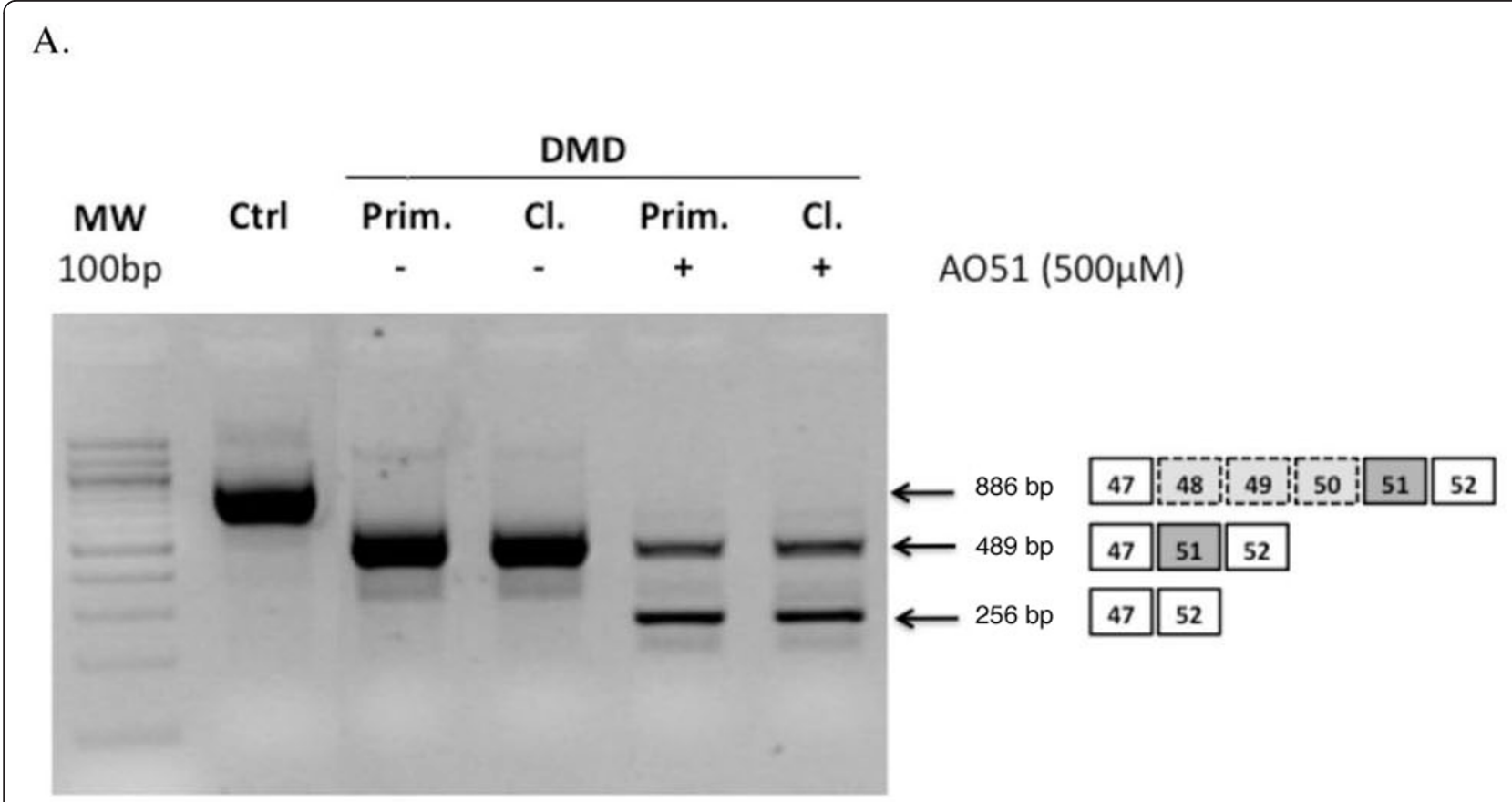

B.

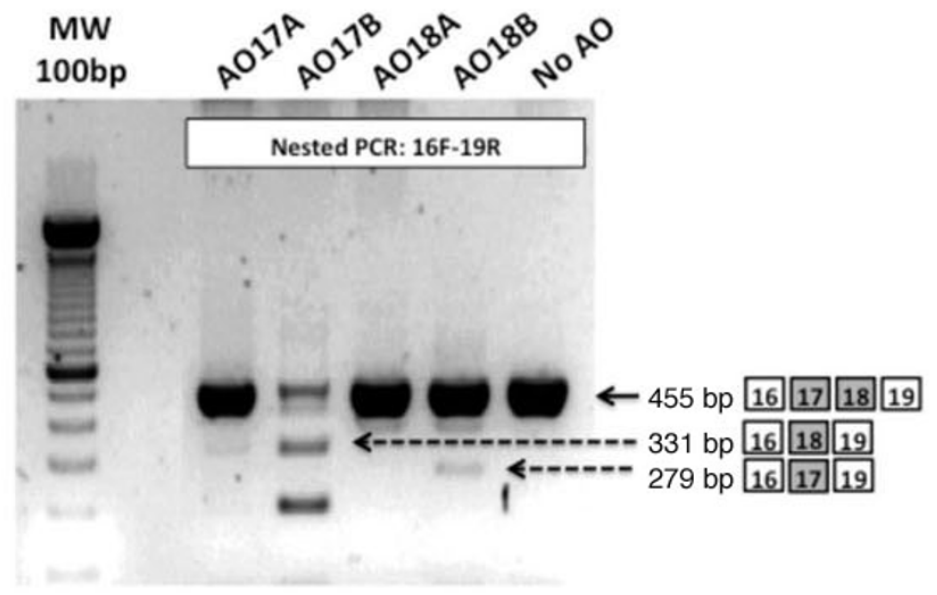

Figure 4 Antisense oligonucleotides (AONs) tested in immortalized cells for exon-skipping pre-clinical studies. (A) Validation of AO51 efficacy for exon 51 skipping on primary (Prim.; Duchenne muscular dystrophy (DMD)) and immortalized cultures of a $\triangle 48-50$ patient with DMD. (B) Evaluation of the efficacy of various AONs on control immortalized cells targeting exons 17 and 18.

generates human myoblasts immortal in culture that maintain myogenic potential both in vitro and in vivo, offering new cell paradigms for pathophysiological studies and novel therapeutic strategies.

\section{List of abbreviations}

AONs: antisense oligonucleotides; CDK-4: cyclin-dependent kinase 4; CMD: congenital muscular dystrophy; DMD: Duchenne muscular dystrophy; DMEM: Dulbecco's modified Eagle's medium; FCS: fetal calf serum; FSHD:

facioscapulohumeral muscular dystrophy; hTERT: human telomerase reverse transcriptase; LGMD2B: limb-girdle muscular dystrophy type 2b; N-CAM: 
neural cell adhesion molecule; OPMD: oculopharyngeal muscular dystrophy; PBS: phosphate-buffered serum; PCR: polymerase chain reaction.

\section{Acknowledgements}

We thank all the patients who provided the biopsies to establish the primary cultures. We also thank the MSG study group, particularly D. Furling for fruitful discussions and L. Dollé, S. Sandal, M. Oloko, S. Vasseur and M. Chapart for technical assistance. We thank Genosafe for help with the transductions and Steve Wilton for providing the antisense oligonucleotides. This work was supported by the MYORES Network of Excellence (contract 511978) and TREAT-NMD (contract LSHM-CT-2006-036825) from the European Commission 6th FP, MYOAGE (contract HEALTH-F2-2009-223576) from the Seventh FP, the ANR Genopath-INAFIB, the ANR MICRORNAS, MyoGrad (GK1631, German Research Foundation), the Duchenne Parent Project Netherlands, CNRS, INSERM, University Pierre and Marie Curie, AFM (Association Française contre les Myopathies) (including network grant \#15123), the Jain Foundation, Parents Project of Monaco, and the European Parent Project.

\section{Author details}

${ }^{1}$ Thérapie des maladies du muscle strié, Institut de Myologie, UM76, UPMC Université Paris 6, Paris, France. ${ }^{2}$ INSERM U974, Paris, France. ${ }^{3}$ CNRS UMR 7215, Paris, France. ${ }^{4}$ Innate Immunity Unit, INSERM U 668, Institut Pasteur, Paris, France. ${ }^{5}$ Service d'Oto-Rhino-Laryngologie et de Chirurgie CervicoFaciale, Faculté de Médecine St Antoine, Université Pierre et Marie Curie, Hôpital Tenon, Paris, France. ${ }^{6}$ The Dubowitz Neuromuscular Centre, Institute of Child Health, University College, London, UK. ${ }^{7}$ Muscle Research Unit, Experimental and Clinical Research Center, Charité University Hospital and Max Delbrück Center for Molecular Medicine, Berlin, Germany. ${ }^{8}$ Faculté de Médecine de Marseille, Université de la Méditerranée, Inserm UMRS 910 Génétique Médicale et Génomique Fonctionnelle, Marseille, France. ${ }^{9}$ Department of Neurology, Hillel Yaffe Medical Center, PO Box 169, Hadera, 38100, Israel. ${ }^{10}$ UT Southwestern Medical Center, Department of Cell Biology, Dallas, TX 75390, USA. ${ }^{11}$ Laboratoire LBCM, Departement de Biologie, Faculté des Sciences, Agadir, Maroc.

\section{Authors' contributions}

$\mathrm{KM}, \mathrm{CT}, \mathrm{AB}, \mathrm{EN}$, and $\mathrm{SC}$ designed and performed the in vitro and in vivo experiments, analyzed data, and wrote the manuscript. AW, PKK, SM, JK, and AA provided technical support. JDS provided the immunodeficient Rag $2^{-/-}$ $\mathrm{YC}^{-1-} \mathrm{C}^{-/-}$mice for the in vivo experiments. JLSG, FM, SP, SS, NL, SB, and TV provided biopsies, and WEW provided DNA constructs. TV and AA discussed the results and gave expert advice. GBB and VM provided conceptual input and supervision. and wrote the manuscript. All authors read and approved the final manuscript.

\section{Competing interests}

The authors declare that they have no competing interests.

Received: 20 June 2011 Accepted: 1 November 2011

Published: 1 November 2011

\section{References}

1. McNally EM, Pytel P: Muscle diseases: the muscular dystrophies. Annu Rev Pathol 2007, 2:87-109.

2. Ruegg MA, Glass DJ: Molecular mechanisms and treatment options for muscle wasting diseases. Annu Rev Pharmacol Toxicol 2011, 51:373-395.

3. Trollet C, Athanasopoulos T, Popplewell L, Malerba A, Dickson G: Gene therapy for muscular dystrophy: current progress and future prospects. Expert Opin Biol Ther 2009, 9:849-866.

4. Trollet C, Anvar SY, Venema A, Hargreaves IP, Foster K, Vignaud A, Ferry A, Negroni E, Hourde C, Baraibar MA, et al: Molecular and phenotypic characterization of a mouse model of oculopharyngeal muscular dystrophy reveals severe muscular atrophy restricted to fast glycolytic fibres. Hum Mol Genet 2010.

5. Vignaud A, Ferry A, Huguet A, Baraibar M, Trollet C, Hyzewicz J, ButlerBrowne G, Puymirat J, Gourdon G, Furling D: Progressive skeletal muscle weakness in transgenic mice expressing CTG expansions is associated with the activation of the ubiquitin-proteasome pathway. Neuromuscul Disord 2010, 20:319-325.
6. Allamand V, Campbell KP: Animal models for muscular dystrophy: valuable tools for the development of therapies. Hum Mol Genet 2000, 9:2459-2467.

7. Mouly V, Aamiri A, Perie S, Mamchaoui K, Barani A, Bigot A, Bouazza B, Francois $V$, Furling $D$, Jacquemin $V$, et al: Myoblast transfer therapy: is there any light at the end of the tunnel? Acta Myol 2005, 24:128-133.

8. Webster C, Blau HM: Accelerated age-related decline in replicative lifespan of Duchenne muscular dystrophy myoblasts: implications for cell and gene therapy. Somat Cell Mol Genet 1990, 16:557-565.

9. Renault V, Thornell LE, Eriksson PO, Butler-Browne G, Mouly V: Regenerative potential of human skeletal muscle during aging. Aging Cell 2002, 1:132-139

10. Wright WE, Shay JW: Historical claims and current interpretations of replicative aging. Nat Biotechnol 2002, 20:682-688.

11. Bodnar AG, Ouellette M, Frolkis M, Holt SE, Chiu CP, Morin GB, Harley CB, Shay JW, Lichtsteiner S, Wright WE: Extension of life-span by introduction of telomerase into normal human cells. Science 1998, 279:349-352.

12. Vaziri $\mathrm{H}$, Benchimol S: Reconstitution of telomerase activity in normal human cells leads to elongation of telomeres and extended replicative life span. Curr Biol 1998, 8:279-282.

13. Zhu CH, Mouly V, Cooper RN, Mamchaoui K, Bigot A, Shay JW, Di Santo JP, Butler-Browne GS, Wright WE: Cellular senescence in human myoblasts is overcome by human telomerase reverse transcriptase and cyclindependent kinase 4: consequences in aging muscle and therapeutic strategies for muscular dystrophies. Aging Cell 2007, 6:515-523.

14. Nowak KJ, Davies KE: Duchenne muscular dystrophy and dystrophin: pathogenesis and opportunities for treatment. EMBO Rep 2004, 5:872-876.

15. Tawil R: Facioscapulohumeral muscular dystrophy. Neurotherapeutics 2008, 5:601-606

16. Brais B, Bouchard JP, Xie YG, Rochefort DL, Chretien N, Tome FM, Lafreniere RG, Rommens JM, Uyama E, Nohira O, et al: Short GCG expansions in the PABP2 gene cause oculopharyngeal muscular dystrophy. Nat Genet 1998, 18:164-167.

17. Bansal D, Miyake K, Vogel SS, Groh S, Chen CC, Williamson R, McNeil PL, Campbell KP: Defective membrane repair in dysferlin-deficient muscular dystrophy. Nature 2003, 423:168-172.

18. Reed UC: Congenital muscular dystrophy. Part I: a review of phenotypical and diagnostic aspects. Arq Neuropsiquiatr 2009, 67:144-168.

19. Bigot A, Klein AF, Gasnier E, Jacquemin V, Ravassard P, Butler-Browne G, Mouly $V$, Furling D: Large CTG repeats trigger p16-dependent premature senescence in myotonic dystrophy type 1 muscle precursor cells. Am J Pathol 2009, 174:1435-1442.

20. Di Donna S, Mamchaoui K, Cooper RN, Seigneurin-Venin S, Tremblay J, Butler-Browne GS, Mouly V: Telomerase can extend the proliferative capacity of human myoblasts, but does not lead to their immortalization. Mol Cancer Res 2003, 1:643-653.

21. Cawthon RM: Telomere measurement by quantitative PCR. Nucleic Acid Research 2002, 30:e47.

22. Cawthon RM: Telomere length measurement by a novel monochrome multiplex quantitative PCR method. Nucleic Acid Research 2009, 37:e21.

23. Negroni E, Riederer I, Chaouch S, Belicchi M, Razini P, Di Santo J, Torrente $Y$ Butler-Browne GS, Mouly V: In vivo myogenic potential of human CD133+ muscle-derived stem cells: a quantitative study. Mol Ther 2009, 17:1771-1778.

24. Cooper RN, Irintchev A, Di Santo JP, Zweyer M, Morgan JE, Partridge TA, Butler-Browne GS, Mouly V, Wernig A: A new immunodeficient mouse model for human myoblast transplantation. Hum Gene Ther 2001, 12:823-831.

25. Cooper RN, Thiesson D, Furling D, Di Santo JP, Butler-Browne GS, Mouly V: Extended amplification in vitro and replicative senescence: key factors implicated in the success of human myoblast transplantation. Hum Gene Ther 2003, 14:1169-1179.

26. Decary S, Mouly V, Hamida CB, Sautet A, Barbet JP, Butler-Browne GS: Replicative potential and telomere length in human skeletal muscle: implications for satellite cell-mediated gene therapy. Hum Gene Ther 1997, 8:1429-1438.

27. Renault V, Piron-Hamelin G, Forestier C, DiDonna S, Decary S, Hentati F, Saillant G, Butler-Browne GS, Mouly V: Skeletal muscle regeneration and the mitotic clock. Exp Gerontol 2000, 35:711-719. 
28. Bigot A, Jacquemin V, Debacq-Chainiaux F, Butler-Browne GS, Toussaint O, Furling $D$, Mouly V: Replicative aging down-regulates the myogenic regulatory factors in human myoblasts. Biol Cell 2008, 100:189-199.

29. Rossi S, Poliani PL, Cominelli M, Bozzato A, Vescovi R, Monti E, Fanzani A: Caveolin 1 is a marker of poor differentiation in Rhabdomyosarcoma. Eur J Cancer 2010.

30. Wang S, Guo L, Dong L, Li S, Zhang J, Sun M: TGF-beta1 signal pathway may contribute to rhabdomyosarcoma development by inhibiting differentiation. Cancer Sci 2010, 101:1108-1116.

31. Stewart N, Bacchetti S: Expression of SV40 large T antigen, but not smal $\mathrm{t}$ antigen, is required for the induction of chromosomal aberrations in transformed human cells. Virology 1991, 180:49-57.

32. Mouly V, Edom F, Decary S, Vicart P, Barbert JP, Butler-Browne GS: SV40 large $T$ antigen interferes with adult myosin heavy chain expression, but not with differentiation of human satellite cells. Exp Cell Res 1996, 225:268-276.

33. Cudre-Mauroux C, Occhiodoro T, Konig S, Salmon P, Bernheim L, Trono D: Lentivector-mediated transfer of Bmi-1 and telomerase in muscle satellite cells yields a duchenne myoblast cell line with long-term genotypic and phenotypic stability. Hum Gene Ther 2003, 14:1525-1533.

34. Stadler G, Chen JC, Wagner K, Robin JD, Shay JW, Emerson CP Jr, Wright WE: Establishment of clonal myogenic cell lines from severely affected dystrophic muscles - CDK4 maintains the myogenic population. Skelet Muscle 2011, 1:12.

35. Perie S, Mamchaoui K, Mouly V, Blot S, Bouazza B, Thornell LE, St Guily JL, Butler-Browne G: Premature proliferative arrest of cricopharyngeal myoblasts in oculo-pharyngeal muscular dystrophy: therapeutic perspectives of autologous myoblast transplantation. Neuromuscul Disord 2006, 16:770-781.

36. Chaouch S, Mouly V, Goyenvalle A, Vulin A, Mamchaoui K, Negroni E, Di Santo J, Butler-Browne G, Torrente Y, Garcia L, Furling D: Immortalized skin fibroblasts expressing conditional $\mathrm{MyoD}$ as a renewable and reliable source of converted human muscle cells to assess therapeutic strategies for muscular dystrophies: validation of an exon-skipping approach to restore dystrophin in Duchenne muscular dystrophy cells. Hum Gene Ther 2009, 20:784-790.

doi:10.1186/2044-5040-1-34

Cite this article as: Mamchaoui et al:: Immortalized pathological human myoblasts: towards a universal tool for the study of neuromuscular disorders. Skeletal Muscle 2011 1:34.

\section{Submit your next manuscript to BioMed Central and take full advantage of:}

- Convenient online submission

- Thorough peer review

- No space constraints or color figure charges

- Immediate publication on acceptance

- Inclusion in PubMed, CAS, Scopus and Google Scholar

- Research which is freely available for redistribution

Submit your manuscript at www.biomedcentral.com/submit 